\title{
Blepharonasofacial malformation syndrome
}

INSERM

\section{Source}

INSERM. (1999). Orphanet: an online rare disease and orphan drug data base.

Blepharonasofacial malformation syndrome. ORPHA:1252

Blepharonasofacial syndrome is a rare otorhinolaryngological malformation syndrome

characterized by a distinctive mask-like facial dysmorphism, lacrimal duct obstruction, extrapyramidal features, digital malformations and intellectual disability. 